\title{
Rapid and Selective Sample Preparation for the Chromatographic Determination of Brassinosteroids from Plant Material Using Solid-Phase Extraction Method
}

\author{
Keiji GamoH*†, Ikuyo YamaguCH* and Suguru TAKATSUTo** \\ *Faculty of Education, Kochi University, Akebono-cho, Kochi 780, Japan \\ **Department of Chemistry, Joetsu University of Education, Joetsu, Niigata 943, Japan
}

\begin{abstract}
This report describes a rapid and selective method of sample preparation for the determination of brassinosteroids in samples of biological origin, based on solid-phase extraction. The steroids, brassinolide and castasterone, which possess a C-22,23-diol group, were retained as their cyclic boronates formed on immobilized phenylboronic acid gel in a mixed solvent of pyridine and acetonitrile. After removal of impurities in the sample, parent brassinosteroids could easily be released from their boronates by a mixed solution of hydrogen peroxide and acetonitrile. The phenylboronic acid phase was shown to have a high affinity for brassinosteroids containing a C-22,23-diol. The present method is a first example for the selective extraction of brassinosteroids in a biological sample using immobilized phenylboronic acid gel.
\end{abstract}

Keywords Brassinosteroid, brassinolide, castasterone, C-22,23-steroidal diol, plant extract, cyclic boronate, immobilized phenylboronic acid gel

Since the first isolation of a steroidal plant-growth promoter, brassinolide ${ }^{1}$, extensive investigation has been carried out to search for new brassinosteroids. More than thirty brassinosteroids have been characterized in the plant kingdom. ${ }^{2}$ The isolation of brassinosteroids from biological material is a lengthy process requiring high skill. Plant extracts often contain large amounts of endogenous contaminants. The amounts of brassinosteroids are in the range $10-100 \mathrm{ng} / \mathrm{g}$, with considerable interference from endogenous materials present in the extracts. Therefore, more extensive sample preparation for plant samples will usually be required.

In early studies on brassinosteroids in plants, solvent partition steps and column chromatography were extensively used. More recently, in many instances it has proved possible to replace such laborious and timeconsuming methods with a solid-phase extraction technique, which is the main subject of this paper. Short cuts in that isolation, taking advantage of special properties of brassinosteroids, would be a considerable help in their isolation, identification and quantitative analysis. One possible strategy would be to exploit the presence of vicinal diol systems found in most biological active brassinosteroids. ${ }^{2,3}$

Boronic acids react selectively with 1,2- and 1,3-diols, $\alpha$-hydroxy acids and other such bi-functional groups to form cyclic derivatives. Brooks ${ }^{4,5}$ has thoroughly explored the use of boronic acids, including phenyl-

† To whom correspondence should be addressed. boronic acid, to give volatile cyclic boronate derivatives of steroids for gas chromatography. We have independently developed the use of cyclic boronates as liquid chromatographic derivatives of brassinosteroids, coupled with the use of the ultraviolet ${ }^{6}$, fluorometric ${ }^{7-9}$, and electrochemical ${ }^{10}$ detectors for selective detection of the boronates.

Recently, immobilized phenylboronic acid (PBA) has become available, attached to either silica, agarose or polymer gel. We have therefore explored the use of solid-phase extraction with columns of immobilized PBA gel for the selective retention of brassinosteroids containing vicinal diol groups.

In this paper the retention and elution characteristics of two typical brassinosteroid compounds, brassinolide and castasterone, on PBA gel have been investigated in order to ascertain the possibilities of this new method of extraction. The use of PBA gel in the extraction of brassinosteroids from plant material has also been attempted to see if the results obtained for standard brassinosteroids can be extended to biological samples.

\section{Experimental}

\section{Chemicals}

All reagents and chemicals were of reagent grade quality or better and were used as purchased without further purification. The water used in the chromatographic mobile phase was doubly distilled. PBA gel, TSK guardgel Boronate-5PW, was purchased from 
TOSO Co., Tokyo. Hydrogen peroxide was obtained from Mitsubishi Gas Industry Co., Kanagawa. Authentic brassinosteroids were synthesized in our laboratory. ${ }^{11,12}$

\section{Solid-phase extraction}

For loading onto the PBA phase, the brassinosteroid standards $(10 \mu \mathrm{g}$ each) and extracts from plants were dissolved in $100 \mu \mathrm{l}$ of a mixed solvent of pyridine/ acetonitrile (1:9). We do not use aqueous alkaline buffers, such as borate and phosphate, for loading these steroids onto the PBA gel, because both they and the plant extracts are not dissolved in those solutions. Each sample was then suspended with PBA gel $(50 \mathrm{mg})$ and heated at $80^{\circ} \mathrm{C}$. The suspension was collected and filtered into a small cartridge by applying a gentle vacuum from a water pump, with the flow rate maintained at $0.5 \mathrm{ml} / \mathrm{min}$.

The PBA gel was then washed with $2 \mathrm{ml}$ fractions of the various buffers and solvent-buffer mixtures. The eluant fractions collected were directly analyzed by reversed-phase HPLC. If some reversed-phase interaction with the PBA gel would be possible, the need for an organic modifier such as methanol or acetonitrile would be considerable. Finally, brassinosteroids adsorbed on PBA gel were released with a mixed solvent $(100 \mu l)$ of acetonitrile and hydrogen peroxide. The contents of the solvents and the temperature effects on releasing steroids were examined.

\section{LC analysis}

A Shiseido Model NANOSPACE liquid chromatograph was employed. A reversed-phase Capcell $\mathrm{Pak}_{18}$ UG 120 column $(1.5 \mathrm{~mm}$ i.d. $\times 25 \mathrm{~cm}$ long, Shiseido, Tokyo) was used at $45^{\circ} \mathrm{C}$. Samples were injected into the column using a Rheodyne Model 7125 rotary valve syringe-loading injector. The optimum mobile phase for the detection and separation of the standard brassinosteroids was a mixture of acetonitrile $/ 20 \mathrm{mM}$ $\mathrm{KH}_{2} \mathrm{PO}_{4}(\mathrm{pH} 4.5)(6: 4)$ and flow rate $0.1 \mathrm{ml} / \mathrm{min}$. The brassinosteroids were monitored by $\mathrm{UV}$ detector at $210 \mathrm{~nm}$. In the case of plant extracts, it was necessary for the released fraction to be evaporated and derivatized with naphthaleneboronic acid to detect trace amounts of these steroids.

\section{Results and Discussion}

Reversed-phase liquid chromatographic data show that the side-chain hydroxyls at C-22 and C-23 can form a five-membered ring in the boronate, but the $\mathrm{C}-2$ and $\mathrm{C}$ 3 hydroxyls of brassinosteroids do not so readily form a cyclic boronate (Fig. 1). ${ }^{13}$ The PBA gel and diol groups for which it has an affinity form a stable cyclic boronate in the presence of basic solvent. Non-bound contaminations are eluted, if necessary, using an organic modifier such as methanol or acetonitrile. The complex is hydrolyzed in an acidic medium and the retained compounds are eluted, again if necessary, with an organic modifier. If this form of extraction could be applied to brassinosteroids, it would provide a more rapid and selective purification method than those presently in use.

Effect of the reaction time for adsorption of the steroids on $P B A$ gel

When loading brassinosteroids to PBA phase, the reaction temperature had a large effect in the range from

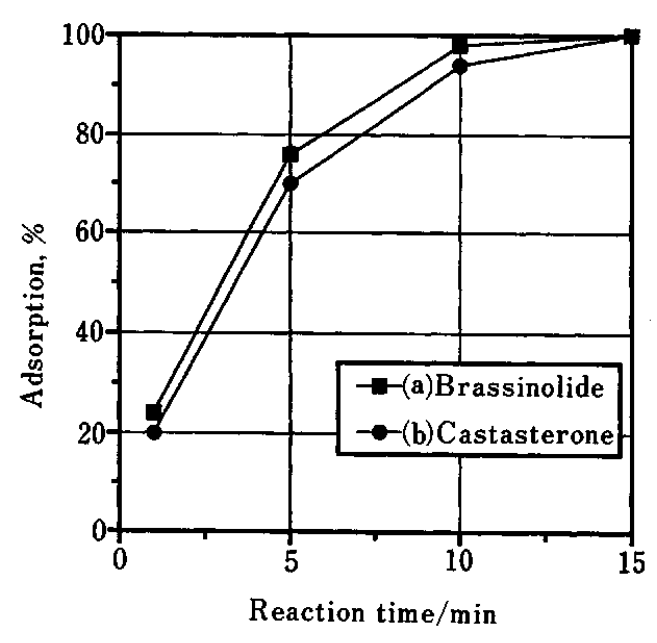

Fig. 2 Time course for adsorption of the steroidal diols on the PBA gel: (a) brassinolide; (b) castasterone.
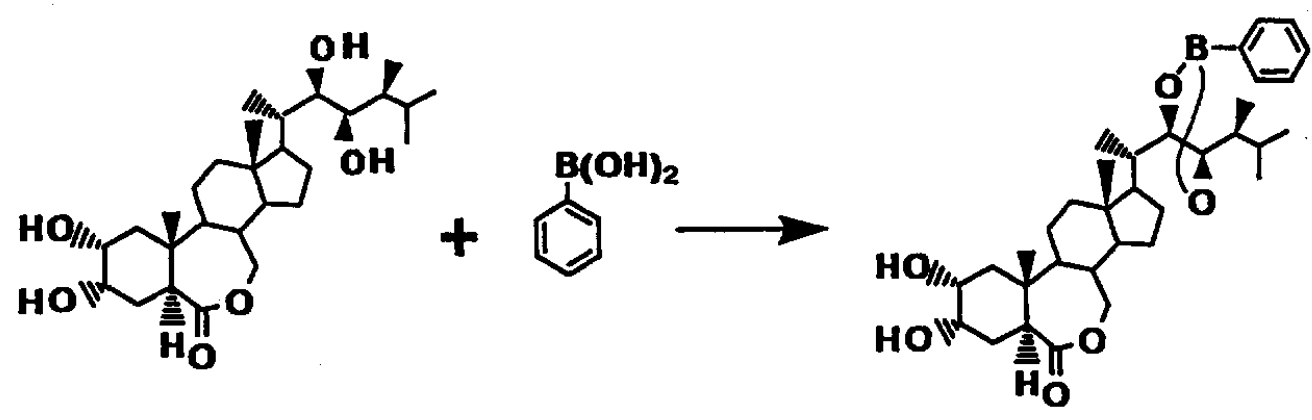

Fig. 1 Structures of brassinolide and the phenylboronate. 
room temperature to $80^{\circ} \mathrm{C}$. The rate of the adsorption was very slow at the room temperature. The time course for the adsorption of the standard steroids on PBA is illustrated in Fig. 2. After heating the suspension at $80^{\circ} \mathrm{C}$ for $15 \mathrm{~min}$, essentially complete retention of the brassinosteroids was observed.

Effect of concentration of hydrogen peroxide on release of the steroids from $P B A$ gel

We had expected that these steroids would be eluted with an acidic eluent, with perhaps the addition of acetonitrile to overcome non-specific secondary interactions. However, washing of the PBA gel with buffers such as phosphate, acetate, formate, $10 \%$ acetic acid, $5 \%$ phosphoric acid, $3 \%$ trifluoroacetic acid and $3 \%$ lactic acid, even with up to $50 \%$ acetonitrile present, showed low recovery yield of parent steroidal compounds.

To obtain a quantitative recovery, we selected hydrogen peroxide since it has been reported ${ }^{14}$ that hydrogen peroxide used for liberating parent diol compounds from their corresponding boronates by oxidative splitting of the $\mathrm{C}-\mathrm{B}$ bond of the boronates. In this work, the effect of concentration of hydrogen peroxide solution used on release of the brassinosteroids from the PBA gel was examined. Figure 3 shows the recovery yield of the steroids in the hydrogen peroxide concentration range $1-10 \%$ when acetonitrile/hydrogen peroxide $(1: 1)$ was used as a releasing solvent. The results indicated that the optimum concentration was $5 \%$.

Effect of concentration of acetonitrile on release of the steroids from $P B A$ gel

We then examined the effect of concentration of

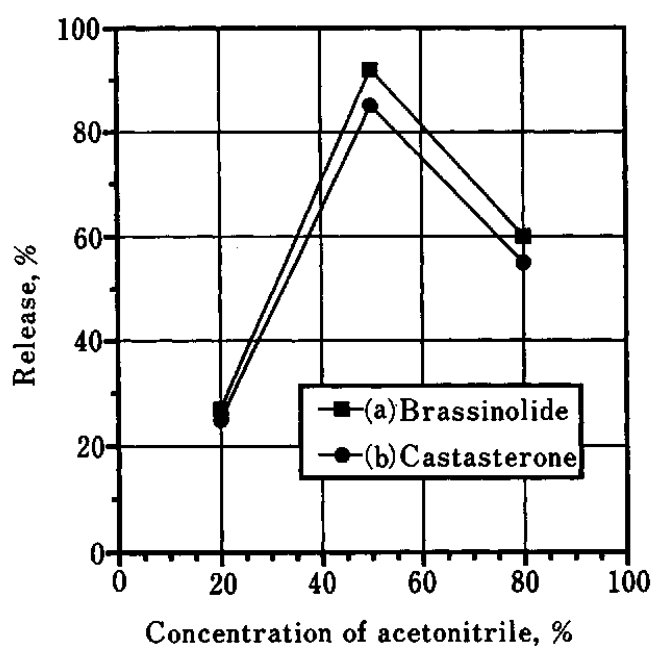

Fig. 4 Effect of concentration of acetonitrile on release of steroidal diols from the PBA gel: (a) brassinolide; (b) castasterone.

acetonitrile used on release of the steroids. Figure 4 shows the recovery yield of the parent steroids in the acetonitrile concentration range $20-80 \%$ when acetonitrile $/ 5 \%$ hydrogen peroxide was used as a releasing solvent. The results indicated that the optimum concentration was $50 \%$.

The releasing yield of the steroids was decreased when a higher concentration of hydrogen peroxide or acetonitrile was used. No plausible explanation is available for the low releasing yield of the steroids. However, it is likely that the optimized concentration of hydrogen

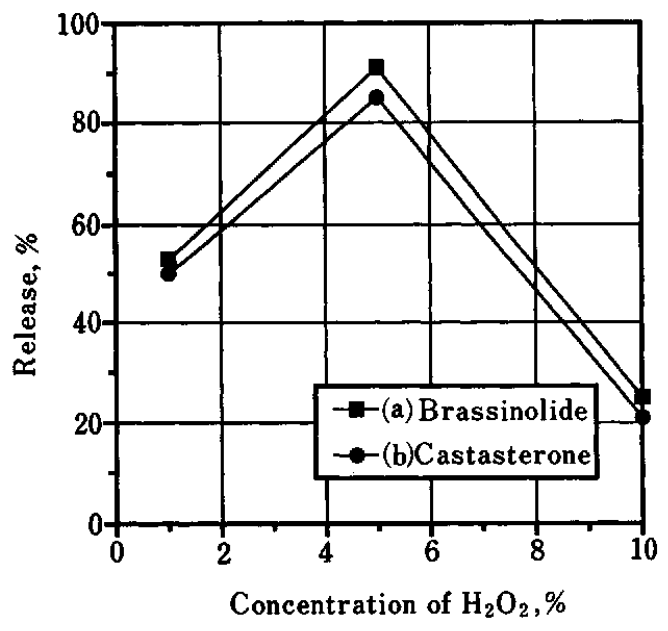

Fig. 3 Effect of concentration of hydrogen peroxide on release of steroidal diols from the PBA gel: (a) brassinolide; (b) castasterone.

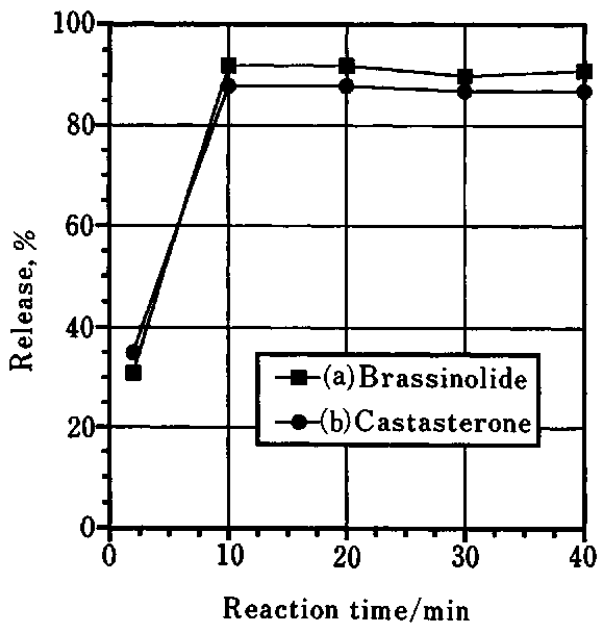

Fig. 5 Time course of recovery yields of the steroidal diols from the PBA gel: (a) brassinolide; (b) castasterone. 
peroxide and acetonitrile plays a $\mathrm{n}$ important role in the hydrolysis of steroidal 22,23-diol boronates.

\section{Effect of reaction time for heating on release of the steroids from PBA gel}

When releasing the steroids adsorbed on PBA gel, the reaction temperature had a slight effect in the range from room temperature to $45^{\circ} \mathrm{C}$. The PBA gel which adsorbed the steroids was suspended with $100 \mu$ l of $50 \%$ acetonitrile in 5\% hydrogen peroxide in a small vial, and then heated at $45^{\circ} \mathrm{C}$. Figure 5 shows the recovery yield of the steroids in the time range 2-40 min after heating. The results indicated that the releasing yield of the steroids has been constant during standing after $10 \mathrm{~min}$ at $45^{\circ} \mathrm{C}$. Typical chromatograms illustrating these results are shown in Fig. 6. The large peaks observed in the void volume are due to solvent mixture and reagent blank.

\section{Application of the proposed method}

In studies on the divided bioactive fraction, which was extracted from bee-collected pollen of broad bean ${ }^{15}$, the

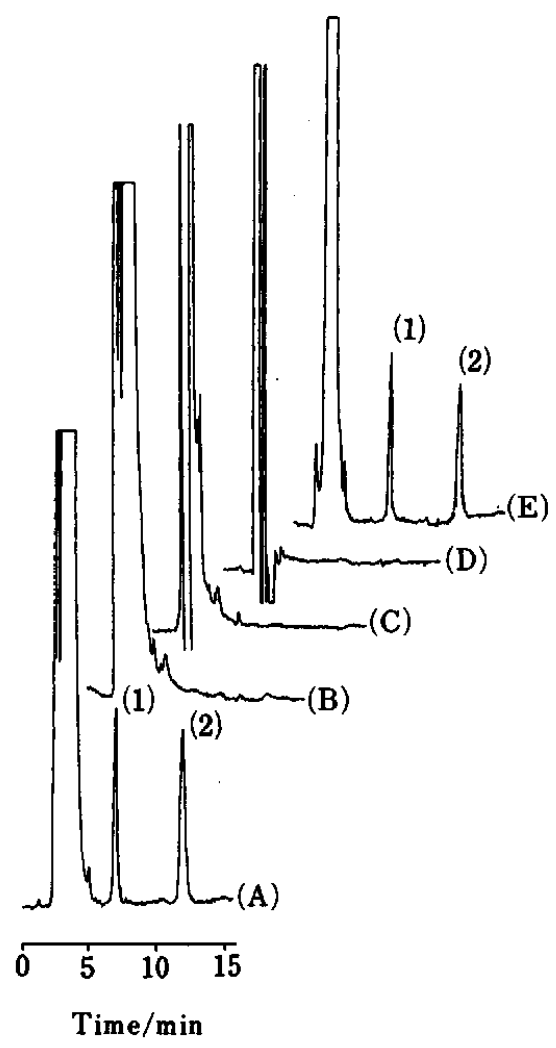

Fig. 6 Chromatograms of standard mixtures of brassinolide and castasterone and the eluent fractions obtained from the PBA gel: (A) standard mixtures of brassinolide and castasterone; (B) sample after loading; (C) after elution with water and then phosphate buffer ( $\mathrm{pH} 2.5$ ); (D) after elution with $50 \%$ acetonitrile in phosphate buffer (pH 2.5); (E) after releasing with $50 \%$ acetonitrile in $5 \%$ hydrogen peroxide. Peaks: (1) brassinolide, (2) castasterone. Conditions are shown in the text.

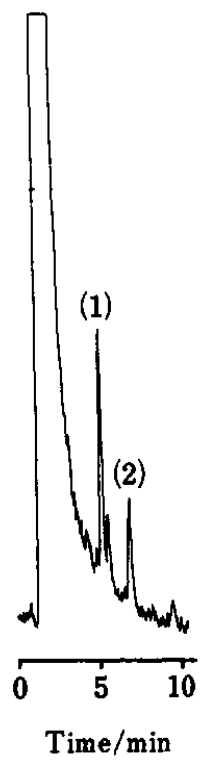

Fig. 7 Chromatogram of the final eluent fraction of plant extract obtained from the PBA gel after derivatization with naphthaleneboronic acid. Peaks: (1) brassinolide, (2) castasterone. Conditions: Capcell Pak $\mathrm{C}_{18}$ UG 120 column (1.5 mm i.d. $\times 25 \mathrm{~cm}$ long); mobile phase, acetonitrile/water (v/v 9:1); flow rate, $0.16 \mathrm{ml} / \mathrm{min}$; temperature, $45^{\circ} \mathrm{C}$; detection, $280 \mathrm{~nm}$.

sample was suspended with the PBA gel in pyridine/ acetonitrile solvent at $80^{\circ} \mathrm{C}$ for $15 \mathrm{~min}$. Washing the gel with acidic buffer, methanol-buffer mixture and acetonitrile eluted contaminants. Brassinosteroids were successively eluted using $50 \%$ acetonitrile in $5 \%$ hydrogen peroxide as a solvent. After evaporating of the release solvent, the residue was derivatized with naphthaleneboronic acid according to our previous method. ${ }^{7}$ The chromatogram illustrating the results is shown in Fig. 7. The large peaks observed in the void volume are due to solvent mixture and excess amounts of naphthaleneboronic acid.

Peak 1 and 2 were identified as those of the brassinolide and castasterone, respectively, by co-chromatography using the corresponding authentic brassinosteroids. The amounts of the steroids in the bioactive fraction were calculated to be $28.4 \mathrm{ng}$ and $19.5 \mathrm{ng}$, respectively, by use of authentic samples for calibration. It was found that purification by solid-phase extraction method using PBA gel of bioactive fraction obtained from preparative TLC was very effective.

In conclusion, the brassinosteroids with a diol group in the C-22,23 are strongly retained on the PBA gel. The present method is the first example for the use of PBA gel in the purification method of brassinosteroids in plants. It is based on the specific formation of stable boronates on PBA gel and the selective release of parent diols from the gel. The method would be very useful for the determination of brassinosteroids in various kinds of biological samples. 


\section{References}

1. M. D. Grove, G. F. Spencer, W. K. Rohwedder, N. Mandava, J. L. Worley, J. D. Warthen, Jr., G. L. Steffens, J. L. Flippen-Anderson and J. C. Cook, Jr., Nature [London], 281, 216 (1979).

2. T. Yokota, Chem. Regul. Plants, 22, 10 (1987).

3. S. Takatsuto, J. Chromatogr., 658, 3 (1994).

4. G. M. Anthony, C. J. W. Brooks, I. Maclean and I. Sangster, J. Chromatogr. Sci., 7, 623 (1969).

5. C. J. W. Brooks and D. J. Harvey, J. Chromatogr., 54, 193 (1971).

6. K. Gamoh, T. Kitsuwa, S. Takatsuto, Y. Fujimoto and N. Ikekawa, Anal. Sci., 4, 533 (1988).

7. K. Gamoh, K. Omote, N. Okamoto and S. Takatsuto, $J$. Chromatogr., 469, 424 (1989).

8. K. Gamoh and S. Takatsuto, Anal. Chim. Acta, 222, 201
(1989).

9. K. Gamoh N. Okamoto, S. Takatsuto and I. Tejima, Anal. Chim. Acta, 228, 101 (1990).

10. K. Gamoh H. Sawamoto, S. Takatsuto, Y. Watabe and H. Arimoto, J. Chromatogr., 515, 227 (1990).

11. S. Takatsuto and N. Ikekawa, J. Chem. Soc., Perkin Trans. $1,1983,2133$.

12. S. Takatsuto and N. Ikekawa, Chem. Pharm. Bull., 30, 4181 (1982).

13. K. Gamoh and C. J. W. Brooks, Anal. Sci., 9, 549 (1993).

14. J. Pis, J. Hykl, M. Budesinsky and J. Harmatha, Collect. Czech. Chem. Commun., 58, 612 (1993).

15. N. Ikekawa, F. Nishiyama and Y. Fujimoto, Chem. Pharm. Bull., 36, 405 (1988).

(Received June 8, 1994)

(Accepted August 24, 1994) 